\title{
Analysis of the principal component algorithm in phase-shifting interferometry
}

\author{
J. Vargas, ${ }^{1, *}$ J. Antonio Quiroga, ${ }^{2}$ and T. Belenguer ${ }^{1}$ \\ ${ }^{1}$ Laboratorio de Instrumentación Espacial, Instituto Nacional de Técnica Aeroespacial, \\ Carretera de Ajalvir Km 4, 28850, Torrejón de Ardoz (Madrid), Spain \\ ${ }^{2}$ Optics Department, Universidad Complutense de Madrid, Facultad de CC. Físicas, \\ Ciudad Universitaria s/n, 28040 Madrid, Spain \\ ${ }^{*}$ Corresponding author: jvargas@fis.ucm.es
}

Received April 13, 2011; revised May 5, 2011; accepted May 5, 2011; posted May 9, 2011 (Doc. ID 145848); published June 7, 2011

\begin{abstract}
We recently presented a new asynchronous demodulation method for phase-sampling interferometry. The method is based in the principal component analysis (PCA) technique. In the former work, the PCA method was derived heuristically. In this work, we present an in-depth analysis of the PCA demodulation method. (C) 2011 Optical Society of America

OCIS codes: $\quad 100.5070,100.2650$.
\end{abstract}

Recently a novel asynchronous phase-shifting method based on principal component analysis (PCA) was presented [1]. PCA is a mathematical procedure that uses an orthogonal transformation to convert a set of observations of possibly correlated variables into a set of values of uncorrelated variables called principal components [2]. In image processing, the PCA algorithm is used to reduce an image set composed by a number of possible correlated images to an uncorrelated image group composed by the principal components [3]. The PCA algorithm is an efficient technique for extracting the uncorrelated information of a given image set.

Suppose that we have $N$ images of size $N_{x} \times N_{y}$. This image set can be expressed as

$$
x=\left[x_{1}, x_{2}, \ldots, x_{N}\right]^{T},
$$

where $x_{n}$ is a column vector with length $M=N_{x} \times N_{y}$ whose elements are taken columnwise from the $n$th image. In Eq. (1), $T$ denotes the transposing operation and $x$ is an $N \times M$ matrix. The first step of the PCA algorithm consists of obtaining the covariance matrix $C$ from $x$ as

$$
C=\left(x-m_{x}\right)(x-m)^{T} .
$$

In Eq. (2), $m_{x}$ has the same size as $x$. All the elements in each column of $m_{x}$ correspond to the mean value of the respective column of $x$. As $C$ is a real and symmetric $N \times$ $N$ matrix, it is always diagonalizable. From matrix theory, the covariance matrix can be diagonalized as

$$
D=U C U^{T},
$$

where $D$ is the diagonalized covariance and $U$ is an orthogonal transformation matrix, both of size $N \times N$. Practically, this diagonalization process can be performed using the singular value decomposition method $[4,5]$. Once the covariance matrix is diagonalized, the principal components are obtained from $U, x$, and $m_{x}$ by

$$
y=U\left(x-m_{x}\right) .
$$

Recently, the PCA algorithm has been used in phaseshifting interferometry (PSI) [1] to obtain the modulating phase of a fringe pattern sequence with no information about the different phase shifts (asynchronous phase detection). In [1], it was shown that the PCA demodulating method is more accurate and fast than the self-calibrating advanced iterative algorithm [6]. However, in [1], the demonstration of the PCA fringe demodulation method was heuristic and no analytical analysis was provided. In this work, we give an analytical demonstration and we show the implicit approximations used by the PCA and their practical consequences.

In PSI, an interferogram sequence can be described using the following expression:

$$
I_{n}(x, y)=S(x, y)+B(x, y) \cos \left(\Phi(x, y)+\delta_{n}\right),
$$

where $I_{n}(x, y), S(x, y)$, and $B(x, y)$ are the intensity map, the background, and the contrast map at pixel $(x, y)$, respectively. $\Phi(x, y)$ is the modulating phase and $\delta_{n}$ is the $n$th phase step. If we rearrange every interferogram as a column vector taken columnwise from the $n$th image, we can rewrite Eq. (ㅁ) as

$$
I_{n, k}=S_{k}+B_{k} \cos \left(\Phi_{k}+\delta_{n}\right),
$$

where $k=[1, \ldots, M]$ is the pixel index and $n=[1, \ldots, N]$ is the image index, with $M$ the total number of pixels and $N$ the number of phase-shifted images. From Eq. (ㅁ) we can form the data matrix introduced in Eq. (1) as

$$
x=\left(\begin{array}{cccc}
S_{1}+B_{1} \cos \left(\Phi_{1}+\delta_{1}\right) & S_{2}+B_{2} \cos \left(\Phi_{2}+\delta_{1}\right) & \ldots & S_{M}+B_{M} \cos \left(\Phi_{M}+\delta_{1}\right) \\
\ldots & \ldots & & \ldots \\
S_{1}+B_{1} \cos \left(\Phi_{1}+\delta_{N}\right) & S_{2}+B_{2} \cos \left(\Phi_{2}+\delta_{N}\right) & \ldots & S_{M}+B_{M} \cos \left(\Phi_{M}+\delta_{N}\right)
\end{array}\right),
$$


where $x_{n}=\left[S_{1}+B_{1} \cos \left(\Phi_{1}+\delta_{n}\right), \ldots, S_{M}+B_{M} \cos \left(\Phi_{M}+\right.\right.$ $\left.\left.\delta_{n}\right)\right]^{T}$ is an $M \times 1$ vector. From $x$ we can obtain the background filtered version as $\tilde{x}=x-m_{x}$. Taking into account that the background is a smooth signal, $m_{x}$ is a good estimation of the background illumination term:

$$
\left\{m_{x}\right\}_{k}=\frac{1}{N} \sum_{n} I_{n, k} \approx S_{k} .
$$

Therefore, $\tilde{x}$ can be expressed as

$$
\begin{aligned}
A & =\left[\begin{array}{lll}
\cos \delta_{1} & \ldots & \cos \delta_{N}
\end{array}\right]^{T}\left[\begin{array}{lll}
\cos \delta_{1} & \ldots & \cos \delta_{N}
\end{array}\right], \\
F & =\left[\begin{array}{llll}
\sin \delta_{1} & \ldots & \sin \delta_{N}
\end{array}\right]^{T}\left[\begin{array}{lll}
\sin \delta_{1} & \ldots & \sin \delta_{N}
\end{array}\right],
\end{aligned}
$$

and $\alpha, \beta$ are the following scalars: $\alpha=\sum_{k=1}^{M} u_{k} u_{k}$ and $\beta=\sum_{k=1}^{M} v_{k} v_{k}$. Matrices $A$ and $F$ come from the product of a vector by itself, so they have rank one. In consequence, they have a single eigenvalue and eigenvector. As the trace is a matrix invariant, the eigenvalues of $A$ and $F$ are given by

$$
\tilde{x}=\left(\begin{array}{cccc}
B_{1} \cos \left(\Phi_{1}+\delta_{1}\right) & B_{2} \cos \left(\Phi_{2}+\delta_{1}\right) & \ldots & B_{M} \cos \left(\Phi_{M}+\delta_{1}\right) \\
\ldots & & & \ldots \\
B_{1} \cos \left(\Phi_{1}+\delta_{N}\right) & B_{2} \cos \left(\Phi_{2}+\delta_{N}\right) & \ldots & B_{M} \cos \left(\Phi_{M}+\delta_{N}\right)
\end{array}\right)
$$

The elements of $\tilde{x}$ can be rewritten as $\tilde{x}_{n, k}=a_{n} u_{k}+b_{n} v_{k}$ with $a_{n}=\cos \delta_{n}, \quad b_{n}=-\sin \delta_{n}, \quad u_{k}=B_{k} \cos \Phi_{k}$, and $v_{k}=B_{k} \sin \Phi_{k}$, and the covariance matrix is given by

$$
C_{i j}=\sum_{k=1}^{M}\left(a_{i} u_{k}+b_{i} v_{k}\right)\left(a_{j} u_{k}+b_{j} v_{k}\right)
$$

Expanding Eq. (10) we have

$$
C_{i j}=\sum_{k=1}^{M} a_{i} a_{j} u_{k} u_{k}+2 a_{i} b_{j} u_{k} v_{k}+b_{i} b_{j} v_{k} v_{k}
$$

If we define $A_{i j}=a_{i} a_{j}, E_{i j}=2 a_{i} b_{j}$, and $F_{i j}=b_{i} b_{j}$, Eq. (11) is rewritten as

$$
C_{i j}=\sum_{k=1}^{M} A_{i j} u_{k} u_{k}+E_{i j} u_{k} v_{k}+F_{i j} v_{k} v_{k} .
$$

If we have more than one fringe in the field of view, Eq. (12) can be simplified making the following approximations:

$$
\begin{aligned}
\sum_{k=1}^{M} u_{k} v_{k} & =\sum_{k=1}^{M} B_{k}^{2} \cos \Phi_{k} \sin \Phi_{k} \ll \sum_{k=1}^{M} u_{k} u_{k} \\
& =\sum_{k=1}^{M} B_{k}^{2} \cos ^{2} \Phi_{k}, \\
\sum_{k=1}^{M} u_{k} v_{k} & =\sum_{k=1}^{M} B_{k}^{2} \cos \Phi_{k} \sin \Phi_{k} \ll \sum_{k=1}^{M} v_{k} v_{k} \\
& =\sum_{k=1}^{M} B_{k}^{2} \sin ^{2} \Phi_{k} .
\end{aligned}
$$

In this case, the covariance matrix is given by

$$
C=\alpha A+\beta F,
$$

where $A$ and $F$ are $N \times N$ matrixes of the form

$$
\lambda_{A}=\sum_{i=1}^{N} \cos ^{2} \delta_{i}, \quad \lambda_{F}=\sum_{i=1}^{N} \sin ^{2} \delta_{i},
$$

and the two unique eigenvectors are

$$
w_{A}=\frac{\left[\cos \delta_{1}, \ldots, \cos \delta_{N}\right]}{\lambda_{A}}, \quad w_{F}=\frac{\left[\sin \delta_{1}, \ldots, \sin \delta_{N}\right]}{\lambda_{F}} .
$$

It is easy to check that both eigenvalues and eigenvectors fulfill $A w_{A}=\lambda_{A} w_{A}$ and $F w_{F}=\lambda_{F} w_{F}$. From Eq. (14), we have that the covariance matrix has rank two and we need to obtain its two eigenvectors. If the phase steps are well distributed in the $[0,2 \pi L]$ range, with $L$ as an integer, we can approximate that

$$
\sum_{n=1}^{N} \cos \delta_{n} \sin \delta_{n} \cong 0,
$$

and, therefore,

$$
A w_{F}=0, \quad F w_{A}=0 .
$$

In this case, the two eigenvectors of the covariance matrix are given by $w_{1}=w_{A}$ and $w_{2}=w_{F}$ with eigenvalues $\lambda_{1}=\alpha \lambda_{A}$ and $\lambda_{2}=\beta \lambda_{F}$. From these eigenvalues and eigenvectors, we can compose the diagonal covariance matrix $D$ and the orthogonal transformation matrix $U$. The unique nonzero values of $D$ will be $D_{11}=\lambda_{1}$ and $D_{22}=\lambda_{2}$. Additionally, the first and second rows of $U$ correspond to $w_{1}$ and $w_{2}$. Once we have obtained the transformation matrix $U$, we can determine the principal components from Eq. (4) from the first and second columns of $y$ as

$$
\begin{aligned}
& y_{1, k}=\sum_{n=1}^{N} B_{k} \cos \left(\Phi_{k}+\delta_{n}\right) \cos \delta_{n}, \\
& y_{2, k}=\sum_{n=1}^{N} B_{k} \cos \left(\Phi_{k}+\delta_{n}\right) \sin \delta_{n} .
\end{aligned}
$$

Following the approximation given by Eq. (18), we obtain

$$
y_{1, k} \cong B_{k} \cos \Phi_{k}, \quad y_{2, k} \cong-B_{k} \sin \Phi_{k} .
$$


Finally, the modulating phase can be obtained as

$$
\Phi_{k}=\arctan \left(\frac{-y_{2, k}}{y_{1, k}}\right)
$$

This result has a very interesting interpretation. Equation (20) is the same result that one will obtain by applying the classical least-squares method [6] with known phase shifts $\delta_{n}$. In our case, the $\delta_{n}$ are obtained from the eigenvectors of the covariance matrix and are given by Eq. (17). From this point of view, the PCA method consists of two steps. First, the phase shifts are estimated from the covariance matrix and, second, the phase is demodulated using the least-squares solution. The analytical derivation depends on the approximations given by Eqs. (8), (13), and (18). In a real experiment, neither of them will be fully accomplished. In this case, the rank of the covariance matrix will be no longer two but something bigger. That means that there will be more than two uncorrelated principal components that will carry information about temporal processes other than the phaseshift variation. As was shown in [1], the typical value for the ratio between the first two eigenvalues and the next is in the range $\left[10^{-2}, 10^{-3}\right]$, indicating the ability of the PCA method to obtain the first two uncorrelated components as given by Eq. (21).

As a summary, we have presented the analytical demonstration of the PCA demodulation method that relies on the three approximations given by Eqs. (8), (13), and (18). First, the temporal average over the interferogram sequence should be a good estimation of the background term. Second, the approximations shown in Eq. (13) have to be fulfilled. These approximations imply that there must be more than one fringe in the interferograms. Finally, Eq. (18) assumes that the different phase shifts must be well distributed in the $[0,2 L \pi]$ interval range with $L$ as an arbitrary positive integer.

\section{References}

1. J. Vargas, J. A. Quiroga, and T. Belenguer, Opt. Lett. 36, 1326 (2011).

2. http://en.wikipedia.org/wiki/Principal_component_analysis.

3. R. C. Gonzalez and R. E. Woods, Digital Image Processing, 3rd ed. (Prentice Hall, 2007).

4. R. Hartley and A. Zisserman, Multiple View Geometry in Computer Vision, 2nd ed. (Cambridge U. Press, 2004).

5. G. H. Golum and C. F. Van Loan, Matrix Computations, 3rd ed. (Johns Hopkins U. Press, 1996).

6. Z. Y. Wang and B. T. Han, Opt. Lett. 29, 1671 (2004). 